\title{
Línea base de la calidad de la mora de castilla (Rubus glaucus) en su cadena alimentaria
}

\section{(Quality baseline of the castilla blackberry (Rubus glaucus) in its food chain)}

\author{
Fernanda Iza ${ }^{1}$, Ximena Rojas-Lema², Yolanda Argüello
}

\begin{abstract}
Resumen:
Una propuesta de mejora en el desempeño de la cadena alimentaria de la mora de castilla (Rubus glaucus) que potencialice su productividad solo puede comenzar desde una línea base o diagnóstico situacional de la calidad de la fruta, donde se identifiquen los principales puntos de mejora. Esta cadena alimentaria identifica cuatro etapas, la cosecha o recolección, poscosecha (almacenamiento y transporte) y comercialización o expendio. El diagnóstico de las etapas comenzó de modo reverso, fue identificado el productor de mayor representatividad y abastecimiento a las comerciantes del punto de expendio. La evaluación de la calidad de la fruta se realizó a través de la caracterización física y química en las cuatro etapas. Se evidenciaron pérdidas de peso o mermas en todas las etapas y ligeros cambios no significativos del color desde rojo azulado tonalidad brillante en la etapa de recolección hasta rojo azulado opaco en la etapa de expendio debido al corto tiempo del ciclo y a las características no climatéricas de la fruta. En todas las etapas ocurrieron cambios significativos en los índices de madurez lo cual significó un incremento de azúcares, disminución del pH e incremento de la acidez titulable. Los resultados señalaron que la fruta cambió sus características fisicoquímicas durante las etapas afectando a la productividad de la misma.
\end{abstract}

Palabras clave: Rubus glaucus, calidad, cadena alimentaria, productividad.

\begin{abstract}
:
A proposal for improvement in the performance of the food chain of castilla blackberry (Rubus glaucus) in order to potentiate their productivity can only start from a baseline or situational diagnosis of the quality of the fruit and hence identify the main points of improvement. The food chain of the fruit identifies three stages, harvest, post-harvest (storage and transport) and marketing or sale. The diagnosis in each stage began with reverse mode. It was identified the most representative producer and the supplying for traders to the point of sale. The quality evaluation of the fruit was performed through chemical and physical characterization in the four stages. Weight loss or losses were evident in all stages, light no significant changes of color from bright red bluish hue in the collection stage until opaque bluish red or off, at the stage of sale due to the short cycle time and the characteristics non-climacteric fruit. However, at all stages of collection, storage, transportation and sale, they presented significant changes in the indices of maturity which meant an increase of sugars, decreased of $\mathrm{pH}$, and increase acidity. The results indicate that the fruit changed its physicochemical characteristics during the stages of the food chain affecting its productivity.
\end{abstract}

Keywords: Rubus glaucus, quality, food chain, productivity

\footnotetext{
${ }^{1}$ Universidad Tecnológica Equinoccial, Quito - Ecuador ( \{farguello, fer88_20\} @ute.edu.ec)

${ }^{2}$ Escuela Politécnica Nacional, Quito - Ecuador (ximena.rojas@epn.edu.ec)
} 


\section{Introducción}

La mora de castilla (Rubus glaucus) es nativa de las zonas tropicales altas de América; se encuentra principalmente en Ecuador, Colombia, Panamá, El Salvador, Honduras, Guatemala, México y Estados Unidos (Franco \& Giraldo, 1999)gran cantidad de vitaminas como la C y E, es una fruta de interés comercial y altamente perecedera, que presenta deterioro debido a su fragilidad e inadecuado manejo en las etapas poscosecha, lo que ocasiona cantidades apreciables de fruta afectadas (Montoya, Londoño, \& Márquez, 2005).

Las etapas de la cadena alimentaria de la fruta son cosecha, recolección, almacenamiento, transporte y comercialización o expendio (Montalvo, 2010). En el Ecuador los principales puntos de comercialización son los mercados, centros donde se expenden alimentos y otros productos al público a través de puestos individuales. De acuerdo con (NTE INEN 2687, 2013), un mercado saludable es un centro de comercialización de alimentos que ha cumplido con todos los requisitos y prácticas para la comercialización y elaboración de alimentos inocuos.

La cadena alimentaria de los productos frescos como la mora de castilla que llegan a comercializarse en los mercados de la ciudad de Quito no es la adecuada, ya que a través de la misma presenta altas pérdidas o mermas de fruta; la calidad de la fruta se ve disminuida con el avance de las etapas y esto se evidencia fundamentalmente en la etapa de expendio. Tanto productores como comerciantes desconocen la importancia del manejo adecuado de la cadena alimentaria de la mora de castilla (Cipagauta \& Loaiza, 2009) y por ende el impacto que este genera.

La productividad en la comercialización de la mora de castilla en el Mercado Iñaquito debido a factores como la baja calidad de la materia prima que arriba al mercado como consecuencia del desconocimiento del manejo y manipulación adecuada de la fruta en las etapas previas y las deficientes condiciones de la infraestructura del mercado que no garantizan la eficiencia de la operación en esta última etapa, ocasionando pérdidas en los ingresos de los comerciantes de esta fruta en el mercado (Cipagauta \& Loaiza, 2009).

De acuerdo con (NTE INEN 2 427, 2010), la calidad de la fruta en condición fresca ya condicionada para su comercialización nacional, toma como referencia requisitos específicos en torno a parámetros físicos y químicos. Los frutos destinados a la comercialización deben presentarse en envases adecuados y el contenido de cada uno debe ser homogéneo, compuesto por frutos del mismo origen, variedad, calibre, calidad y un estado de coloración y madurez homogéneo, de acuerdo con la variedad. En la Tabla 1, se presenta el calibre de la fruta, en (a) con referencia a la Norma Técnica Ecuatoriana (NTE INEN 2 427, 2010), y en (b) con base en la Norma Técnica Colombiana (NTC 4106, 1997). 
Tabla 1. Calibre de la fruta

\begin{tabular}{ccc} 
Calibre & $\begin{array}{c}\text { Diámetro } \\
(\mathbf{m m})\end{array}$ & $\begin{array}{c}\text { Longitud } \\
(\mathbf{m m})\end{array}$ \\
\hline Grande & $>25$ & $>25$ \\
Mediano & $25-18$ & $25-20$ \\
Pequeño & $<18$ & $<20$ \\
\hline
\end{tabular}

(a) (NTE INEN 2427: 2010)

\begin{tabular}{ccc}
\hline Calibre & Diámetro(mm) & $\begin{array}{c}\text { Peso } \\
\text { promedio } \\
(\mathbf{g})\end{array}$ \\
\hline A & $>=27$ & 9.2 \\
B & $26-23$ & 8.8 \\
C & $22-19$ & 6.2 \\
D & $18-14$ & 4.2 \\
E & $<=13$ & 3.2 \\
\hline
\end{tabular}

(b) (NTC 4106, 1997)

La medición del color en las frutas y vegetales frescos contribuye a la evaluación de la calidad de estos por la fuerte relación con cambios químicos como el índice de madurez, o el efecto de factores externos como la temperatura y la humedad en las diferentes etapas poscosecha.

El color en las frutas frescas se debe a la presencia de distintos compuestos, principalmente pigmentos orgánicos naturales (Badui, 2006), muchos de los cuales tienen una función biológica importante en el tejido; entre la estructura básica del color se tiene (1) estructuras químicas derivadas de los núcleos tetrapirrólicos: la clorofila y su función fotosintética, (2) estructuras químicas derivadas del isopreno: carotenos y xantofilas, (3) estructuras químicas derivadas del benzopirano: antocianinas y flavonoides, (4) estructuras químicas derivadas de las betacianinas de color rojo y betaxantinas de color amarillo. La composición química y la estructura del material determinan si la luz puede reflejarse, absorberse o transmitirse y su importancia relativa determina la apariencia del alimento, donde el factor más importante es la reflexión especular y difusa que definen el brillo y el tono respectivamente (Retting \& Ah-Hen, 2014).

La medición del color puede ser realizada de forma visual a través de una codificación numérica para cada estado de madurez, como se muestra en la Figura 1 en base a los requisitos de (NTE INEN 2 427, 2010), o instrumental a través de colorímetros triestímulo (colorímetros Hunter Lab) o a través de coordenadas rectangulares adimensionales $\left(L^{*}, a^{*}, b^{*}\right)$ junto con otro de coordenadas cilíndricas $\left(\mathrm{L}^{*}, \mathrm{H}^{*}, \mathrm{C}^{*}\right)$ y visión digital. El profesor Albert Mushell estableció la ciencia del color y la definió técnicamente con 3 atributos: matiz (tono, tinte, color "hue"), croma (saturación, intensidad, pureza "chroma”) y brillo (brillante, brillo “brigthness") (Retting \& Ah-Hen, 2014).

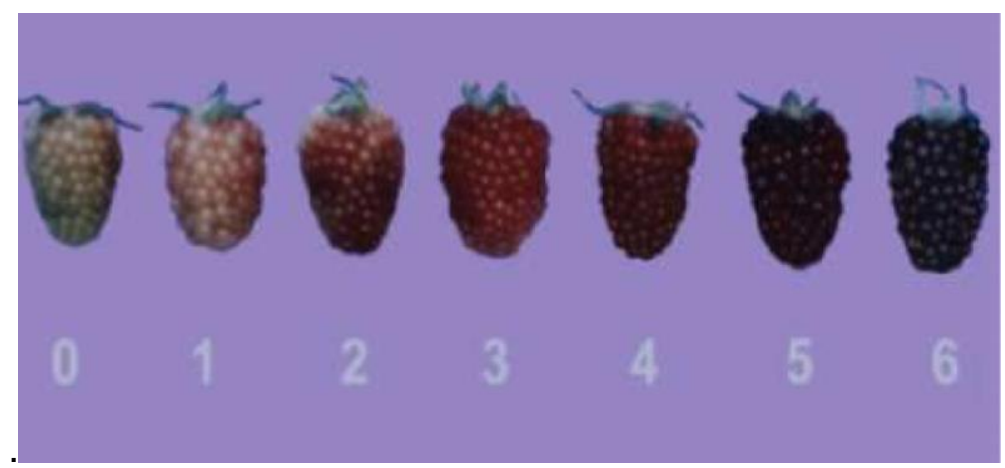

Figura 1. Escala de color de la mora de castilla (NTC 4106, 1997 ; NTE INEN 2 427, 2010) 
Un indicador de calidad del proceso a lo largo de la cadena alimentaria es la productividad, donde un factor fundamental es la evaluación de pérdidas o mermas que se presenta en cada etapa, mediante la variación de peso de la fruta. Este es un indicativo del nivel de conocimiento en el manejo adecuado de la fruta.

Finalmente, de acuerdo con (NTE INEN 2 427,2010), el cumplimiento de los requisitos químicos de la fruta señalan los criterios de calidad y seguridad alimentaria en base al contenido de sólidos solubles ( ${ }^{\circ}$ Brix), acidez titulable (ácido cítrico \%) e índice de madurez. La Tabla 2, presenta los valores mínimos y máximos de estos requisitos.

Tabla 2. Requisitos químicos de la mora de castilla

\begin{tabular}{|c|c|c|c|}
\hline & \multicolumn{2}{|c|}{$\begin{array}{l}\text { MADUREZ DE } \\
\text { CONSUMO }\end{array}$} & \multirow[t]{2}{*}{$\begin{array}{l}\text { METODO DE } \\
\text { ENSAYO }\end{array}$} \\
\hline & Mín & Máx & \\
\hline \multicolumn{4}{|l|}{ Mora variedad Castilla } \\
\hline Acidez titulable \% (acido cítrico) & - & 1,8 & NTE INEN 381 \\
\hline Sólidos solubles totales, ${ }^{\circ}$ Brix & 9,0 & - & NTE INEN 380 \\
\hline Índice de madurez ${ }^{\circ} \mathrm{Bx} /$ acidez titulable & 5,0 & - & Ver 8.2 \\
\hline \multicolumn{4}{|l|}{ Mora variedad Brazos } \\
\hline Acidez titulable \% (acido cítrico) & - & 2,1 & NTE INEN 381 \\
\hline Sólidos solubles totales, ${ }^{\circ} \mathrm{Brix}$ & 7,0 & - & NTE INEN 380 \\
\hline Índice de madurez ${ }^{\circ} \mathrm{Bx} /$ acidez titulable & 3,3 & - & Ver 8.2 \\
\hline
\end{tabular}

(NTE INEN 2 427, 2010)

\section{Metodología}

En la presente investigación se realizó el estudio de las características físico-químicas de frutos de mora de castilla (Rubus glaucus) proveniente de la provincia de Carchi, cantón Huaca cuyo destino de expendio fue el mercado Iñaquito de Quito. Este análisis tomó como referencia la (NTE INEN 2 427, 2010) en las cuatro etapas de la cadena de la fruta, para esto se inició con la codificación de cada etapa: recolección $(R)$, almacenamiento $(A)$, transporte $(T)$ y comercialización o expendio (E). A continuación se determinó un plan de muestreo con base en las generalidades de la fruta y el sector de procedencia. Inicialmente se evaluó exclusivamente la etapa $\mathrm{R}$ y las diferencias significativas de las propiedades físicas (calibre y color) de la fruta de 3 cosechas diferentes $\mathrm{C} 1, \mathrm{C} 2$ y $\mathrm{C} 3$ en fechas y horarios coordinados conjuntamente con el proveedor de la misma; posteriormente se evaluaron las diferencias significativas de las propiedades físicas (calibre y color) y químicas (índice de madurez) de la fruta de las 3 cosechas C1, C2, y C3 y su efecto al pasar por las etapas de $R, A, T, y E$ de la cadena alimentaria.

\subsection{Análisis físico}

El análisis físico comenzó en la etapa $\mathrm{R}$ al determinar el calibre de la fruta a través de la medición de las dimensiones: diámetro, longitud y peso, y verificar el nivel de madurez a través del color mediante la escala visual detallada en la (NTE INEN 2 427, 2010). 
Para medir el diámetro y la longitud se utilizó un calibrador pie de rey, con una precisión de 0.05 mm; para determinar el peso se usó una balanza semianalítica Pioneer Ohaus $(0.001 \mathrm{~g})$ donde se registró el peso individual (una a una) de $1 \mathrm{Kg}$ de mora conforme lo señala la (NTE INEN 1750, 1994) de muestreo de hortalizas y frutas frescas.

Para la determinación objetiva de las diferencias del color en las etapas $R, A, T$ y $E$ de la cadena se empleó el colorímetro de superficie marca Konica Minolta, modelo Chroma Meter CR400; utilizando la escala de color CIE L*, $a^{*}, b^{*}$ y empleando las ecuaciones 1 y 2 (Montalvo, 2010; Retting \& Ah- Hen, 2014).

$$
\begin{aligned}
& \mathrm{H}^{*}=\tan ^{-1} \frac{\mathrm{b}}{\mathrm{a}} \\
& \mathrm{C}=\left(a^{* 2}+b^{* 2}\right)^{\frac{1}{2}}
\end{aligned}
$$

$\mathbf{a}^{*}=$ Intensidad de color rojo/verde (+a indica rojo, -a indica verde)

$\mathbf{b}^{*}=$ Intensidad de color amarillo/azul (+b indica amarillo, -b indica azul)

$\mathbf{C}=$ intensidad (croma) .

$\mathbf{H}^{*}=$ Tono (hue), varía entre 0 y $360^{\circ}$

El croma $C$ toma el valor 0 para estímulos acromáticos y normalmente no pasa de 150 y su color va desde los grises pálidos apagados a los más fuertes y vivos. (Retting \& Ah-Hen, 2014).

\subsection{Análisis químicos}

Para efectuar los análisis químicos se determinó el tamaño de la muestra, constituido por $1 \mathrm{~kg}$ de fruta licuada y cernida, se realizó un muestreo al azar en cada etapa de la cadena alimentaria, según lo establecido en la norma para muestreo de hortalizas y frutas frescas (NTE INEN 1750, 1994).

El análisis químico determinó el grado de madurez de la fruta, donde se evaluaron los requisitos de acidez titulable, sólidos solubles e índice de madurez. Para el caso de la acidez titulable la medición fue de acuerdo con el ácido predominante, que para la mora de castilla corresponde al ácido cítrico; se pesó $30 \mathrm{~g}$ de pulpa y se diluyó en $200 \mathrm{ml}$ con agua destilada, se tomó una alícuota de $20 \mathrm{ml}$ y se tituló con $\mathrm{NaOH} 0.1 \mathrm{~N}$, hasta el $\mathrm{pH}$ de 8.2 que es el punto de viraje del indicador fenolftaleína, se registró la lectura del gasto, según lo descrito por la A.O.A.C. (2007) y con la ecuación 3 se obtuvo el valor buscado.

$$
\% \text { Ác. Cítrico }=\left(\frac{\mathrm{V}_{\mathrm{NaOH}} * \mathrm{~N} * \mathrm{Meq} * \mathrm{~V}_{\mathrm{t}}}{\mathrm{P}_{\mathrm{m}} * \mathrm{~V}_{\mathrm{a}}}\right) * 100
$$

Dónde:

$\mathrm{V} \mathrm{NaOH}=$ Volumen de hidróxido de sodio consumidos en la titulación (ml)

$\mathrm{N}=$ Normalidad del hidróxido de sodio

Meq = Miliequivalentes del ácido cítrico (0.064)

$\mathrm{Vt}=$ Volumen final $(\mathrm{ml})$ 
$\mathrm{Pm}=$ Peso de la muestra $(\mathrm{g})$

$\mathrm{Va}=$ Volumen de la alícuota $(\mathrm{ml})$

Para la determinación del contenido de sólidos solubles se colocó una gota de la pulpa de la mora sobre el prisma de la superficie de un refractómetro calibrado de marca Milwaukee de 0 a $85 \%$ y se registró la medida en Brix, según lo descrito por la A.O.A.C. (2007).

Para el análisis de $\mathrm{pH}$ se empleó un potenciómetro con electrodo previamente calibrado con buffer a $\mathrm{pH} 7$ y $\mathrm{pH}$ 4. Se tomaron $20 \mathrm{ml}$ de la pulpa, se introdujo el electrodo en el centro de la muestra con agitación constante y se registró la lectura, según la A.O.A.C. (2007).

Para determinar el índice de madurez se utilizó la ecuación 4.

$$
\text { Índice de Madurez }=\frac{\text { Sólidos Solubles Totales }\left({ }^{\circ}\right. \text { Brix) }}{\text { Acidez Titulable (Ác.Cítrico \%) }}
$$

\subsection{Diseño experimental y Análisis estadístico}

Para la evaluación de la calidad de la fruta en la etapa de $\mathrm{R}$ se utilizó un diseño unifactorial completamente aleatorizado de la variable cosecha $C$ en 3 niveles $C 1, C 2$ y $C 3$, en donde se analizó la influencia de las mismas sobre el calibre a través del diámetro, longitud y peso. Los resultados fueron procesados mediante un análisis de varianza ANOVA multifactorial y las medias fueron comparadas a través de la prueba de DMS a un nivel de significancia de 0.05 , con el uso del programa estadístico Statgraphics Centurión XV.

Adicionalmente se utilizó un diseño experimental $A \times B$; la variable $A$ la etapa de la cadena alimentaria en 4 niveles $R, A, T, C$ y la variable $B$ la cosecha en 3 niveles $C 1, C 2$ y C3, en donde se analizó la influencia de las mismas sobre la pérdida de peso, el color, el pH, la acidez titulable, los sólidos solubles, e índice de madurez. Los resultados fueron procesados mediante un análisis de varianza ANOVA multifactorial y las medias fueron comparadas a través de la prueba de Tukey a un nivel de significancia de 0.05, con el uso del programa estadístico Statgraphics Centurión XV.

\section{Resultados}

\subsection{Análisis físicos}

Los resultados de las mediciones del calibre promedio de la fruta en la etapa $R$ para las 3 cosechas de estudio se presentan en la Tabla 3.

Tabla 3. Calibre de la fruta

\begin{tabular}{|c|c|c|c|c|c|c|c|}
\hline Etapa & Muestra & $\begin{array}{c}\text { Diametro } \\
(\mathrm{mm})\end{array}$ & $\begin{array}{c}\text { Diámetro* } \\
(\mathrm{mm})\end{array}$ & $\begin{array}{c}\text { Longitud } \\
(\mathrm{mm})\end{array}$ & $\begin{array}{c}\text { Longitud }{ }^{*} \\
(\mathrm{~mm})\end{array}$ & Peso (g) & 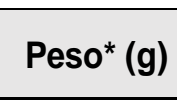 \\
\hline \multirow{3}{*}{$\mathbf{R}$} & C1 & $22.7 \mathrm{a}$ & \multirow{3}{*}{$22.76 \pm 0.90$} & $27.9 \mathrm{a}$ & \multirow{3}{*}{$27.76 \pm 0.23$} & $9.91 \mathrm{a}$ & \multirow{3}{*}{$9.03 \pm 1.53$} \\
\hline & C2 & $23.7 \mathrm{a}$ & & $27.9 \mathrm{a}$ & & $9.93 \mathrm{a}$ & \\
\hline & C3 & $21.9 \mathrm{a}$ & & $27.5 \mathrm{a}$ & & $7.26 \mathrm{a}$ & \\
\hline
\end{tabular}

${ }^{*}$ media \pm DS $(n=30)$

Letras minúsculas iguales significa que no hay diferencias estadísticas significativas del calibre entre cosechas. Empleando prueba Tukey 5\% 


\subsection{Color}

En la Figura 2, se presenta el análisis de la luminosidad, intensidad de color y matiz en cada etapa.

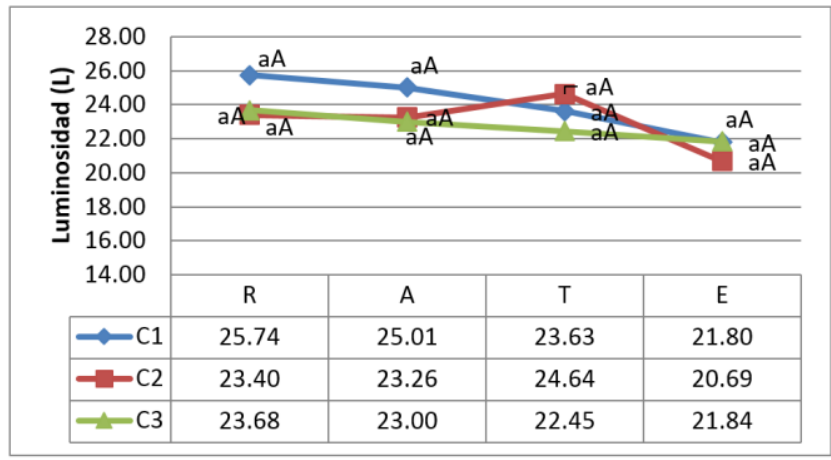

(a)

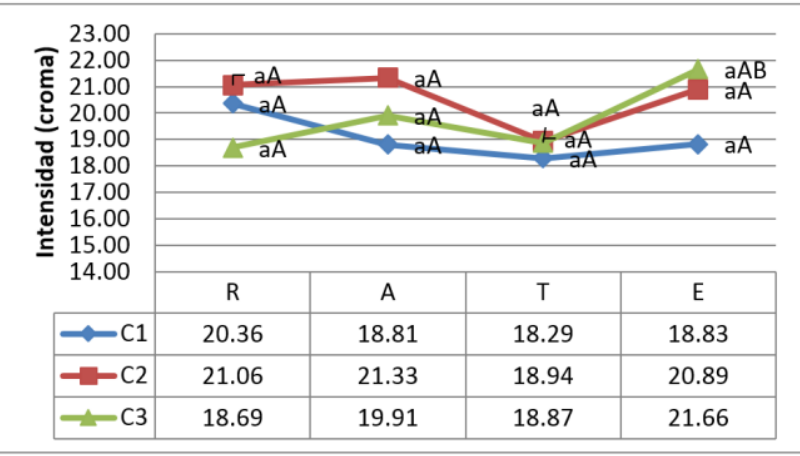

(b)

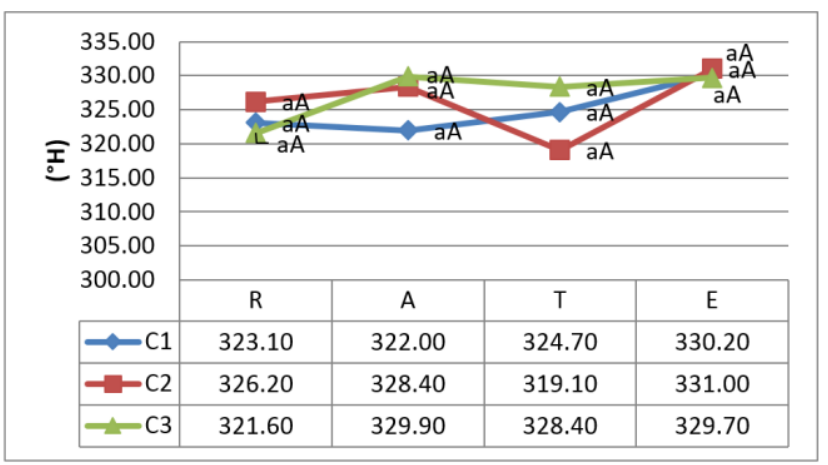

(c)

Figura 2. Escala de color de la mora de castilla (a) Luminosidad, (b) Intensidad de color y (c) matiz o tono.

\subsection{Pérdidas de peso}

En la Tabla 4, se presenta el análisis de las pérdidas de peso en las etapas de la cadena en cada cosecha evaluada.

Tabla 4. Pérdida de peso en las etapas de las 3 cosechas con sus diferencias significativas

\begin{tabular}{|c|c|c|c|c|c|c|c|}
\hline \multicolumn{8}{|c|}{ Pérdida de peso $\%{ }^{1,2}$} \\
\hline Día & $\begin{array}{l}\text { Tiempo } \\
\text { (h) }\end{array}$ & $\begin{array}{c}\text { Temperatura } \\
\left({ }^{\circ} \mathrm{C}\right)\end{array}$ & Etapas & C1 & C2 & C3 & $\begin{array}{c}\text { Pérdida peso*/ } \\
\text { Etapa }\end{array}$ \\
\hline 1 & 0 & $12-13$. & $\mathrm{R}$ & $0.00 \mathrm{Aa}$ & $0.00 \mathrm{Aa}$ & $0.00 \mathrm{Aa}$ & $0.00 \pm 0.0$ \\
\hline 2 & 17 & $9-11$ & $\mathrm{~A}$ & $2.50 \mathrm{Bb}$ & $1.25 \mathrm{Bb}$ & $1.25 \mathrm{Bb}$ & $1.67 \pm 0,72$ \\
\hline 2 & 21 & $13-20$ & $T$ & $3.85 \mathrm{Cc}$ & $3.80 \mathrm{Cc}$ & $3.80 \mathrm{Cc}$ & $3.81 \pm 0.03$ \\
\hline 2 & $24-25$ & $20-24$ & $\mathrm{E}$ & $5.33 \mathrm{Dd}$ & $5.26 \mathrm{Dd}$ & $6.57 \mathrm{Dd}$ & $5.72 \pm 0.74$ \\
\hline \multicolumn{4}{|c|}{ Pérdida peso*/Cosecha } & $2.92 \pm 2.26$ & $2.58 \pm 2.39$ & $2.91 \pm 2.91$ & \\
\hline & & & ey $(p<0.05)$ & & 1.268 & & 0.297 \\
\hline
\end{tabular}

${ }^{*}$ media \pm DS $(n=30)$

${ }^{1}$ Letras minúsculas distintas en una misma columna denotan diferencias estadísticas significativas de la pérdida de peso entre las etapas y letras iguales no hay diferencias. Empleando prueba Tukey p<0.05.

2 Letras mayúsculas distintas en una misma fila denotan diferencias estadísticas significativas de la pérdida de peso entre las cosechas y letras iguales no hay diferencias. Empleando prueba Tukey $p<0.05$.

\subsection{Análisis químico}

En la Figura 3 se presentan los resultados alcanzados de $\mathrm{pH}$, sólidos solubles ( ${ }^{\circ}$ Brix), acidez titulable e índice de madurez de la mora de castilla en cada etapa poscosecha. 


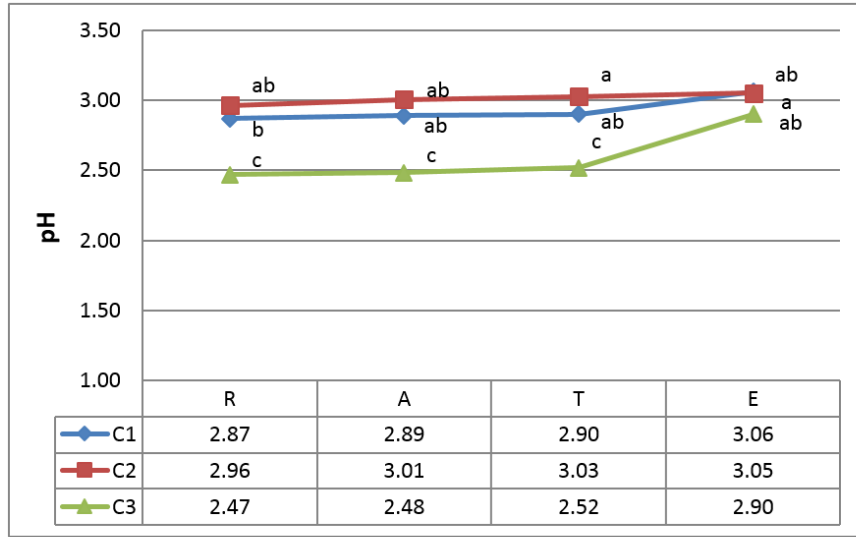

(a)

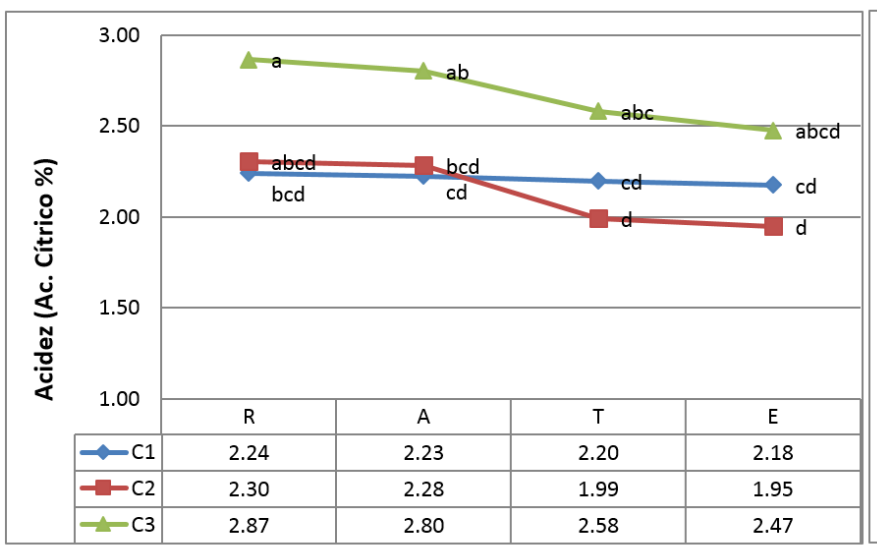

(c)

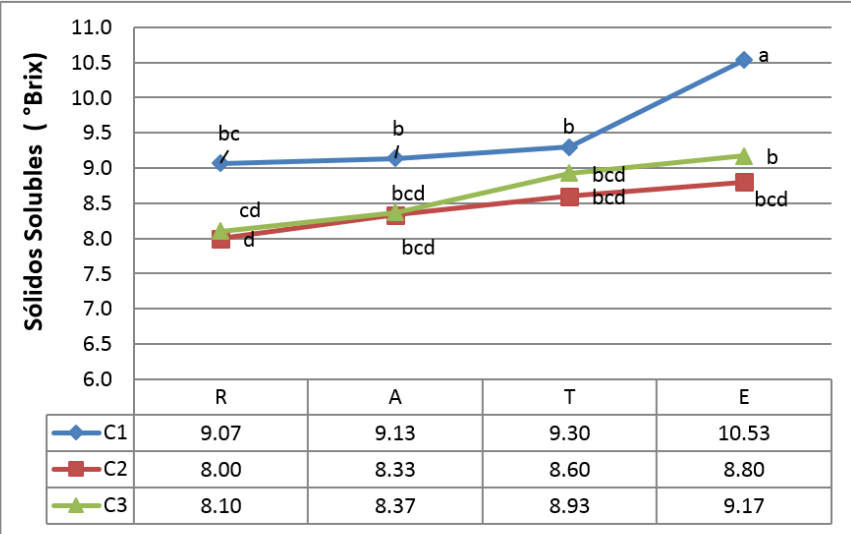

(b)

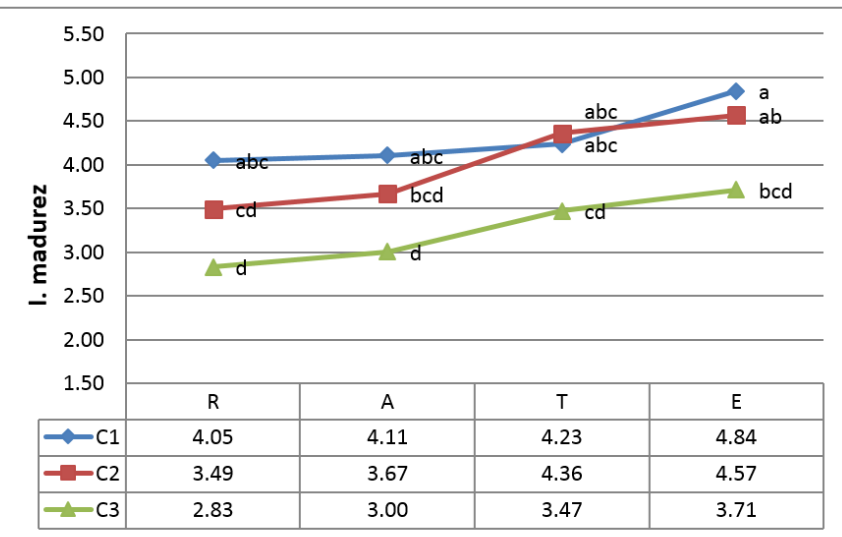

(d)

Figura 3. (a) pH, (b) Sólidos solubles ('Brix), (c) Acidez titulable (Ac cítrico \%), (d) Índice de madurez

\section{Discusión}

\subsection{Análisis Físicos}

Al analizar el calibre de la fruta en la etapa $R$, se registraron valores de peso promedio: $9.03 \pm$ $1.53 \mathrm{~g}$ y dimensiones promedio de diámetro: $22.76 \mathrm{~mm}$ y longitud: $27.76 \mathrm{~mm}$ que señalaron un calibre entre mediano (25 - $18 \mathrm{~mm}$ ) a grande > $25 \mathrm{~mm}$ de acuerdo con la (NTE INEN 2 427, 2010), y calibre A longitud $\geq 27 \mathrm{~mm}$ y peso 9.2 g según (NTC 4106, 1997), similares resultados reportó (García \& García, 2001). Según menciona (Montalvo, 2010), en sus investigaciones sobre la mora de castilla, las mejores accesiones ecuatorianas seleccionadas por peso fueron de 7.04 a $7.36 \mathrm{~g}$, pesos menores a los registrados en este estudio, por lo que se verificó son mejores accesiones debido tipo de suelo y condiciones edafológicas del cultivo en la localidad de Huaca en la provincia del Carchi.

En la etapa $R$, el productor de la fruta no espera que esta llegue a su correcta madurez. Un adecuado proceso de recolección que permita reducir las pérdidas y conservar la calidad requiere un recolección de fruta con un estado de madurez homogéneo (García \& García, 2001). Con respecto a la figura 1 debería estar entre los grados 3 y 4 .

No se evidenciaron cambios significativos del color de la mora desde la etapa $R$ hasta la etapa $E$ debido a las características no climatéricas de la fruta. 
En relación con el color, un valor medio de cromaticidad $a^{*}$ positivo no significativo $(p<0.05)$ desde 14.39 a 18.65 basándonos en la escala croma de color, se puede indicar que la mora registró un incremento desde el color rojo claro a rojo oscuro, mientras que con el valor de cromaticidad y $b^{*}$ positivo no significativo $(p<0.05)$, con valores entre 2.97 y 12.26 que indican colores desde amarillo a rojo azulado. El ángulo Hue $(\stackrel{\circ}{H})$ señaló la tonalidad con valores entre 0 y $360^{\circ}$ que indicaron tonalidades de rojo oscuro; en la cromaticidad $C^{*}$ cuya escala puede ir de 0 a 150. se obtuvieron valores no significativos $(p<0.05)$, entre 18.28 a 21.66 que correspondieron a colores rojo opacos o apagados, debido a la maduración y evaporación; valores similares para $a^{*}, b^{*}, \stackrel{\circ}{H}$, y $\mathrm{C}^{*}$ fueron reportados por Montalvo (2010), en una mora con el $50 \%$ y $75 \%$ de madurez de nivel 4 a 5 de la escala de color visual de los requisitos de la (NTE INEN 2 427, 2010). Los valores de $L^{*}$ registraron cambios no significativos $(p<0.05)$ entre 25.7 y de 21.8 en las etapas $R$ y $E$ respectivamente, lo que significó mayor oscurecimiento en los frutos, similares resultados reportó (Farinango, 2010).

Las antocianinas son pigmentos que dan el color rojo, violeta y azul a la mayoría de las frutas. Una de las características en la maduración de las frutas rojas es la sustitución del color verde inicial por el rojo o violeta por la síntesis de pigmentos coloreados (Farinango, 2010; García \& García, 2001). Según (Delgado, 2012), indica que las antocianinas presentan una fuerte actividad antioxidante que ejerce una protección contra los radicales libres y también presenta un efecto de inhibición frente a las enzimas oxidativas. Además, las antocianinas son susceptibles a los cambios de $\mathrm{pH}$.

Según (Bejarano, 1992) en la mora el índice de madurez utilizado para el consumo en fresco se relaciona con el viraje de color a negro morado brillante y para el estado de madurez comercial que es la que busca el productor se recomienda cosecharla con una coloración rojo escarlata uniforme.

\subsection{Análisis químico}

La Figura 3 (a) muestra que en las 3 cosechas los valores de $\mathrm{pH}$ presentaron un ligero aumento, desde la etapa R: $2.77 \pm 0.24$ hasta la etapa E: $2.99 \pm 0.10$ aunque sin diferencias significativas $(p<0.05)$. Este ascenso se debió a que hubo un aumento de temperatura, en las etapas $\mathrm{T}$ y $\mathrm{E}$. Farinango (2010), también reporta una tendencia ascendente del pH en la fruta en su madurez organoléptica, con un valor promedio de 2.79 .

Reina (1998) en sus investigaciones poscosecha con la mora reportó un incremento del pH desde 2.98 a 3.18, es decir menor acidez, atribuyó este resultado a la temperatura de almacenamiento que superó los $8 \stackrel{\circ}{\circ}$. Con esto, es necesario tener en cuenta que esta fruta tiene gran susceptibilidad a ser almacenada a altas temperaturas por lo cual debe evitarse que la fruta esté a temperaturas mayores de $10^{\circ} \mathrm{C}$ ya que se incrementa el porcentaje de deterioro de la fruta. A 
mayor temperatura mayor pérdida de agua, el deterioro de la apariencia de la fruta es causado por el etileno, e incremento de la tasa de respiración que promueve el crecimiento de patógenos y acelera los procesos de senescencia. Según (Freire, 2012), la fruta recién cosechada presentó un $\mathrm{pH}$ promedio de 2.85 y se observó una ligera tendencia ascendente cuando llegaron a los sitios de comercialización estos fueron transportados en caja de cartón de $4 \mathrm{~kg}$, tomando en cuenta que el transporte fue de Ambato hasta Quito.

No se observaron diferencias significativas del $\mathrm{pH},(\mathrm{p}>0.05)$ de la fruta en la etapa $\mathrm{R}: 2.77 \pm 0.24$ a $2.99 \pm 0.10$ en la etapa $E$; Montalvo (2010), reportó una tendencia similar en la cual observó que la mora con una madurez del $75 \%$ de viraje de color (de rojo a negro) se produce el incremento de 2.78 a 2.89 y de 2.56 a 2.88 en dos accesiones de mora de castilla respectivamente. (Farinango, 2010), también reportó una tendencia ascendente del pH en la fruta en su madurez organoléptica, con un valor promedio de 2.79 .

El incremento de $\mathrm{pH}$ también pudo deberse a la distancia entre la zona productora y el sitio de expendio, así como las condiciones ambientales como temperatura, esto se relaciona con lo mencionado por (Reina, 1998), que a mayor temperatura, en los primeros días de la poscosecha, es mayor la variación en los parámetros químicos ya que esta fruta tiene gran susceptibilidad a ser almacenada a temperaturas altas mayores a $8^{\circ} \mathrm{C}$, en lo que refiere a la duración de la vida útil del producto.

En la Figura 3 (b) se pudo observar que en las 3 cosechas los valores de sólidos solubles se incrementan desde $8.39 \pm 0.55^{\circ}$ Brix, en la etapa $R$ hasta $9.50 \pm 0.85^{\circ}$ Brix, en la etapa $E$, comportamiento que coincide con lo observado por (Dayron, Fischer, \& Flórez, 2006; Montalvo, 2010; García, 2008 y Reina, 1998), para los primeros días de almacenamiento de la fruta.

Además se observó que los contenidos de sólidos solubles fueron superiores a los publicados en la Norma Técnica Colombiana NTC 4106, 1997 de $8.50{ }^{\circ}$ Brix para la mora de castilla madura, pero similares a los reportados por Montalvo (2010) que van desde 9 hasta $11^{\circ}$ Brix. Según (Reina, 1998), los cambios del contenido de sólidos solubles Brix en los días poscosecha presentaron una tendencia ascendente a temperatura ambiente.

Según Montalvo (2010), los sólidos solubles se incrementaron con la maduración, no solo por el incremento en el contenido de azucares, sino también por causa de la deshidratación junto al incremento de los pigmentos propios de la fruta, a lo que se añade un aumento de la concentración de los mismos.

Como se observa en la Figura 4, en las 3 cosechas se registró una disminución no significativa $(p<0.05)$ de la acidez titulable promedio por etapa desde $2.47 \pm 0.33 \%$ en $\mathrm{C}$, hasta $2.20 \pm 0.28 \%$ en $\mathrm{E}$, esto sucedió porque la mora en las etapas $\mathrm{T}$ y $\mathrm{C}$ aumentaron su tasa respiratoria, y su contenido de azúcares. Un comportamiento similar reportó (Montalvo, 2010), la acidez tiende a 
disminuir con la maduración y senescencia debido al aumento de la tasa respiratoria y el contenido de azúcares y pigmentos.

En la Figura 3 (c) se puede observar que en las 3 cosechas existió un aumento del índice de madurez desde la etapa $R$ con $3.46 \pm 0.57^{\circ}$ Brix / Ácido cítrico \% hasta la etapa $\mathrm{E}$ con $4.37 \pm 0.61$ ${ }^{\circ}$ Brix / Ácido cítrico \% con diferencias significativas entre las etapas de R, A, T y E respectivamente. Esto se debe a que mientras más tiempo transcurre, la fruta madura más. Según (Reina, 1998), a medida que el fruto madura, la acidez baja, entre tanto que el porcentaje de azúcares aumenta.

Con el análisis de sólidos solubles se puede determinar que existen diferencias significativas entre la etapa $R$ y $E$ debido a que se analizó la fruta luego de las 24 horas transcurridas entre la recolección y el expendio en el mercado; se evidenció que la fruta no fue recolectada con una correcta madurez.

La acidez titulable no presentó diferencias significativas, pero sí un descenso. Se observó que en la etapa $E$ para las 3 cosechas la mora de castilla existió una tendencia descendente en relación con la obtenida en la etapa R. Según lo reportado por el autor (Reina, 1998), la acidez tiende a reducir con la maduración y senescencia debido al aumento de la tasa respiratoria y el contenido de azúcares y pigmentos.

El Índice de madurez aumentó ligeramente desde la etapa $R$ hasta $E$, esto se debió a que en esta última etapa la fruta estuvo sometida a una temperatura mayor a los $23^{\circ} \mathrm{C}$, también se pudo ver que no existieron diferencias significativas entre las etapas.

Los sólidos solubles ${ }^{\circ}$ Brix, $\mathrm{pH}$, acidez titulable e índice de madurez variaron en cada cosecha, pudo deberse a muchos factores como son temporada de cosecha, factores ambientales, temperatura, humedad, y factores precosecha por esta razón se pudo observar varianza entre algunos análisis, debido a que resulta complicado determinar cuáles fueron los factores precosecha que influyen en estas diferencias no se profundizó en el estudio de estas diferencias. Además cada planta regularmente tiene frutos de diferente grado de madurez y se cosechó de distintas plantas en distintos espacios de terreno.

\section{Conclusiones y Recomendaciones}

En la etapa de recolección es importante poner atención en el tamaño o calibre de la mora ya que esto contribuye a una clasificación más uniforme de la fruta, basado en indicadores de calidad bajo aspectos físicos.

Con el análisis químico realizado se determinó que en las etapas de transporte y comercialización existió un incremento de los sólidos solubles, pH e índice de madurez, con respecto a la acidez esta disminuyó conforme pasa el tiempo, esto se debe a que la fruta se encontraba a 
temperaturas mayores a los $10^{\circ} \mathrm{C}$, incrementando la tasa de respiración que aceleraron los procesos de senescencia.

Un incremento de los pigmentos de la fruta no solo se debe a la maduración sino también a la pérdida de humedad por el calor de la respiración, lo cual significa un incremento de la concentración de azúcares a causa de la deshidratación.

En la cadena alimentaria de la mora de castilla se determinó que la etapa de recolección es la más importante y delicada ya que la fruta debe cosecharse con un adecuado grado de madurez comercial que en la escala (NTE INEN 2 427, 2010; INCONTEC, 1997) está entre 3 y 4 (algunas drupas amarillas y rojo escarlata brillante), esto definirá la calidad y resistencia de la fruta para las siguientes etapas.

El análisis estadístico realizado con respecto a las pérdidas de calidad demostró que en la etapa de expendio existió mayor porcentaje de pérdidas de hasta del 6\%; esto se debió a los tiempos de procesos de valor no agregado durante 24 horas desde la etapa de recolección, sumado a la falta de control de temperatura durante las etapas y uso empaques inadecuados.

Se recomienda realizar estudios de estandarización peso y volumen máximo adecuado por envase en la etapa de recolección de la mora de castilla para evitar pérdidas de peso por presión y escurrimiento de la fruta de la base del envase.

Se recomienda el control de la temperatura $(4-8)^{\circ} \mathrm{C}$ en las etapas de la cadena alimentaria de la mora de castilla identificadas para este ciclo de gestión del negocio.

\section{Bibliografía}

Badui, S. (2006). Química de los Alimentos (4ta Ed. ed.). México, México: Pearson.

Bejarano, W. (1992). Promoción de exportaciones agrícolas no tradicionales (PRO EXANT). Manual de Mora (Rubus glaucus Benth). Quito, Ecuador.

Cipagauta, D., \& Loaiza, C. (2009). Tesis para obtener el título de Ingeniera Industrial. Mejoramiento de los procesos productivos y caracterización de la cadena de abastecimiento para los concesionarios de la plaza de mercado Alameda de la ciudad de Cali. Cali, Colombia.

Dayron, A., Fischer, G., \& Flórez, R. (2006). Almacenamiento refrigerado de frutos de mora de Castilla (Rubus glaucus Benth) en empaques con atmósfera modificada. Agronomía colombiana.

Delgado, F. (2012). Manejo orgánico del cultivo de mora (Rubus sp.). Cuenca, Ecuador. 
Farinango, M. (2010). Tesis. Estudio de la fisiología poscosecha de la mora de castilla (Rubus glaucus Benth) y de la mora variedad brazos (Rubus sp.). Quito, Ecuador.

Franco, G., \& Giraldo, M. (1999). El cultivo de la mora. Pereira, Colombia.

Freire, V. (2012). Tesis para obtener el título de Ingeniero de Agroindustrial. Universidad Politécnica N. Alternativas de mejora en el manejo poscosecha y comercialización de la mora de castilla (Rubus glaucus B) proveniente de la provincia de Tungurahua. Quito.

García , M., \& García, H. (2001). Manual de CORPOICA Manejo Cosecha y Poscosecha de mora, lulo y tomate de árbol. Colombia.

INCONTEC. (1997). Norma técnica colombiana NTC 4106. Colombia: INCOTEC.

Montalvo, D. (diciembre de 2010). Tesis. Evaluación de la calidad poscosecha de las accesiones seleccionadas de mora de castilla (Rubus glaucus Benth) provenientes de las provincias de Tungurahua y Bolívar. Proyecto previo a la obtención del título de Ingeniero Agroindustrial. Quito.

Montoya, A., Londoño, J., \& Márquez, C. (2005). Blackberry liquor (Rubus glaucus Benth) with different pulp percentages. Medellín.

NTC 4106. (1997). Norma Técnica Colombiana NTC 4106:1997. COLOMBIA: NTC.

NTE INEN 1750. (1994). Norma Técnica Ecuatoriana NTE INEN 1750:1994. QUITO: INEN.

NTE INEN 2 427. (2010). Norma Técnica Ecuatoriana NTE INEN 2 427: 2010. Quito: INEN.

NTE INEN 2 427. (2010). Norma Técnica Ecuatoriana NTE INEN 2 427:2010. QUITO: INEN.

NTE INEN 2687. (2013). Norma Tecnica Ecuatoriana NTE INEN 2687:2013. QUITO: INEN. Recuperado el 10 de noviembre de 2015, de http://normaspdf.inen.gob.ec/pdf/nte/2687.pdf.

Reina, C. (1998). Proyecto de titulación previo a la obtención del título de Ingeniero Agrónomo. Universidad Sur colombiana. Manejo poscosecha y evaluación de la calidad para la mora de castilla (Rubus Glaucus) que se comercializa en la ciudad de Neiva. Neiva, Colombia.

Retting, M. K., \& Ah - Hen, K. (2014). El color de los alimentos un criterio de calidad medible. Agrosur(DOI 104206). 